\title{
No significant effect of cinacalcet on cardiovascular outcomes in patients undergoing dialysis-EVOLVE results
}

In a recent study published in The New England Journal of Medicine and presented at ASN Kidney Week 2012, the EVOLVE trial investigators report that the calcimimetic agent, cinacalcet, has no statistically significant effect on cardiovascular outcomes or death in patients with secondary hyperparathyroidism undergoing dialysis.

\section{4 ...the trial should be interpreted as nondefinitive 77}

Secondary hyperparathyroidism is very common in patients with chronic kidney disease and might contribute to their increased risk of cardiovascular events and death because of its potential role in vascular calcification. EVOLVE-a prospective, multicentre, randomized, placebo-controlled trial in which an ethnically diverse cohort of 3,883 adults with moderate to severe secondary parathyroidism on haemodialysis were enrolled-was designed to test the hypothesis that cinacalcet therapy might reduce the risk of death or nonfatal cardiovascular events in dialysis-dependent patients.

In their unadjusted intention-to-treat analysis, the investigators found a $7 \%$ reduction in the risk of the primary composite end point of time to death or first nonfatal cardiovascular event (myocardial infarction, hospitalization for unstable angina, heart failure or a peripheral vascular event) among patients treated with cinacalcet (median exposure 21.2 months) and conventional therapy (including phosphate binders and/or vitamin D sterols) compared with those who received placebo (median exposure 17.5 months) and conventional therapy. However, this decrease in risk was not statistically significant.

After adjusting for baseline characteristics, including age and history of cardiac disease, there was a $12 \%$ reduction in the risk of the primary composite end point in the cinacalcet group compared with the placebo group. The investigators describe this reduction as "nominally significant". Cinacalcet therapy substantially reduced the rates of severe, unremitting hyperparathyroidism and parathyroidectomy but was associated with a sevenfold increase in the risk of hypocalcaemia. There were no significant differences in fracture rates between the cinacalcet and placebo groups.

As the prespecified, unadjusted analysis showed a nonsignificant beneficial effect of cinacalcet on cardiovascular events and death, the investigators suggest that "the trial should be interpreted as nondefinitive."

Ellen F. Carney

Original article The EVOLVE Trial Investigators. Effect of cinacalcet on cardiovascular disease in patients undergoing dialysis. N. Engl. J. Med. doi:10.1056/NEJMoa1205624 